\title{
Local delivery of minocycline-loaded PEG-PLA nanoparticles for the enhanced treatment of periodontitis in dogs
}

This article was published in the following Dove Press journal:

International Journal of Nanomedicine

18 August 2014

Number of times this article has been viewed

\author{
Wenxin Yao' \\ Peicheng $\mathrm{Xu}^{\prime}$ \\ Zhiqing Pang ${ }^{2,3}$ \\ Jingjing Zhao ${ }^{2,3}$ \\ Zhilan Chai ${ }^{2,3}$ \\ Xiaoxia $\mathrm{Li}^{4}$ \\ Huan $\mathrm{Li}^{2,3}$ \\ Menglin Jiang 2,3 \\ Hongbo Cheng 2,3 \\ Bo Zhang ${ }^{2,3}$ \\ Nengneng Cheng ${ }^{4}$ \\ 'Shanghai Xuhui District Dental \\ Center, ${ }^{2}$ Key Laboratory of Smart \\ Drug Delivery, Ministry of Education, \\ ${ }^{3}$ Department of Pharmaceutics, \\ School of Pharmacy, Fudan University, \\ ${ }^{4}$ Department of Pharmacology, \\ School of Pharmacy, Fudan University, \\ Shanghai, People's Republic of China
}

Correspondence: Peicheng Xu

Shanghai Xuhui District Dental Center,

685 Zhaojiabang Road, Shanghai 200032,

People's Republic of China

Tel +86 2I 64037289

Fax +86 21 346। 1287

Emailxpc1238@|26.com

Zhiqing Pang

Key Laboratory of Smart Drug Delivery,

Ministry of Education, Department of

Pharmaceutics, School of Pharmacy,

Fudan University, 826 Zhangheng Road,

Shanghai 201203, People's Republic of

China

Tel +86 2I 51980069

Fax +86 2I 5I 980069

Email zqpang@fudan.edu.cn
Background: Rapid local drug clearance of antimicrobials is a major drawback for the treatment of chronic periodontitis. In the study reported here, minocycline-loaded poly(ethylene glycol)poly(lactic acid) nanoparticles were prepared and administered locally for long drug retention and enhanced treatment of periodontitis in dogs.

Methods: Biodegradable poly(ethylene glycol)-poly(lactic acid) was synthesized to prepare nanoparticles using an emulsion/solvent evaporation technique. The particle size and zeta potential of the minocycline-loaded nanoparticles (MIN-NPs) were determined by dynamic light scattering and the morphology of the nanoparticles was observed by transmission electron microscopy. The in vitro release of minocycline from MIN-NPs and in vivo pharmacokinetics of minocycline in gingival crevice fluid, after local administration of MIN-NPs in the periodontal pockets of beagle dogs with periodontitis, were investigated. The anti-periodontitis effects of MIN-NPs on periodontitis-bearing dogs were finally evaluated.

Results: Transmission electron microscopy examination and dynamic light scattering results revealed that the MIN-NPs had a round shape, with a mean diameter around $100 \mathrm{~nm}$. The in vitro release of minocycline from MIN-NPs showed a remarkably sustained releasing characteristic. After local administration of the MIN-NPs, minocycline concentration in gingival crevice fluid decreased slowly and retained an effective drug concentration for a longer time (12 days) than Periocline ${ }^{\circledR}$. Anti-periodontitis effects demonstrated that MIN-NPs could significantly decrease symptoms of periodontitis compared with Periocline and minocycline solution. These findings suggest that MIN-NPs might have great potential in the treatment of periodontitis.

Keywords: minocycline, nanoparticles, periodontitis, local delivery

\section{Introduction}

"Periodontitis" is a common chronic disease that leads to the destruction of toothsupporting tissues, absorption of alveolar bone, and, finally, tooth loss. ${ }^{1,2}$ Considerable research has focused on the etiology of human periodontal disease, ${ }^{3}$ and it has been generally accepted that chronic periodontitis is induced by microorganisms. Periodontal pockets provide a moist, warm, nutritious, and anaerobic environment that profits microbial colonization and multiplication. ${ }^{4}$ Previous research demonstrated that only 10 to 30 bacteria species, mainly Gram-negative anaerobic bacteria, live here, ${ }^{5}$ but it has now been found that approximately 500 bacterial taxa sojourn here. ${ }^{4}$ The amounts and species of the bacteria are variable in different parts of the biofilm and depend on the effectiveness of oral hygiene procedures, depth of pocket, flow of gingival crevice fluid (GCF), type of interacting microbes, and so on. ${ }^{6,7}$ A recent study has indicated that periodontal disease not only affects human oral health but may also induce several systemic diseases. ${ }^{8}$ 
The fundamental treatment of periodontitis is to reduce the pathogenic bacteria by instrumental debridement, ${ }^{9}$ such as ultrasonic scaling and root planning. However, instrumental debridement cannot completely remove pathogens that have invaded soft tissue and some anatomically inaccessible areas such as furcation areas and root depressions. ${ }^{10}$ In view of this, antimicrobial therapy by antibiotics is often executed as adjuvant treatment soon after instrumental debridement. ${ }^{11}$ In order to achieve effective inhibitory concentration in the GCF of the periodontal pockets, large doses must be taken, which may lead to associated side effects and resistance of antibiotics. ${ }^{12}$ Therefore, localized drug-delivery systems containing antibacterial agents have been developed, including fibers, strips, films, implants, gels, and so forth, and now many products are commercially available. ${ }^{13-15}$ These are administered in the periodontal pocket after ultrasonic scaling and root planning to enhance the local effect. Compared with the conventional systemic administration of antibiotics, these new formulations reduce the frequency of administration and keep the drug concentrations within the desired range. Further, the side effects are reduced significantly. ${ }^{3}$ However, some issues still exist. The vehicles of fibers and strips must be removed after complete release of the drug by professionals, and the wound is not conducive to recovery. ${ }^{16}$ The application of gels is more convenient than fibers and strips, but the issues are burst release and the rapid local drug clearance of antimicrobials. ${ }^{3}$

Biodegradable nanoparticles (NPs) have been developed rapidly over the past few years since they have shown great potential in drug delivery. ${ }^{17}$ NPs have more advantages than other new formulations and many of the already-mentioned problems can be avoided. NPs can offer sustained release of drugs and have the ability to adsorb onto polymer gels and act as a glue to bond pieces of hydrogels and tissues together. ${ }^{18}$ Based on these properties, NPs can adhere to diseased tissues longer and maintain local drug concentration for a long time. Due to the small particle size, NPs are able to penetrate into alveolar bone trabeculae, the underlying connective tissue, and even the periodontal pocket areas below the gum, ${ }^{14,19,20}$ which can also significantly improve the antibacterial effect. Successful periodontal treatment requires a high initial antibiotic concentration followed by the continued release of the drug at a lower concentration, both of which can be realized with NPs. ${ }^{21}$

"Minocycline" belongs to the tetracycline family, but it has a broader antibacterial spectrum than other tetracyclines and can be applied in the treatment of periodontitis. ${ }^{21,22}$ It has been demonstrated that minocycline can affect the immune response caused by cell factors and have a beneficial effect on periodontal health. ${ }^{23}$ In this study, minocycline was encapsulated in poly(ethylene glycol)-poly(lactic acid) (PEG-PLA) NPs, which are metabolized to nontoxic $\mathrm{CO}_{2}$ and $\mathrm{H}_{2} \mathrm{O}$ in the body, ${ }^{24}$ and the pharmacokinetics and pharmacodynamics of this formulation were investigated through a periodontitis model in beagle dogs.

\section{Materials and methods Materials}

Methoxy-poly(ethylene glycol) (MPEG; molecular weight [MW] 3,000 Da) was supplied by NOF Corporation (Tokyo, Japan) and dried under vacuum in a desiccator with $\mathrm{P}_{2} \mathrm{O}_{5}$ overnight before polymer synthesis. D, L-lactide (purity: 99.5\%) was purchased from Purac Biochem (Gorinchem, The Netherlands) and purified by twice recrystallizing in dried ethyl acetate. Stannous octoate $\left[\mathrm{Sn}(\mathrm{Oct})_{2}\right](95 \%$ pure; Sigma-Aldrich, St Louis, MO, USA) was distilled under vacuum and was used by dissolving it in dry toluene. Minocycline hydrochloride (MIN) and tetracycline (both 99.5\% pure) were obtained from the HuBei Xing Galaxy Chemical Corporation Ltd. (Wuhan, People's Republic of China). MIN ointment (Periocline ${ }^{\circledR}$ ) was purchased from Sunstar Inc. (Osaka, Japan). Double distilled water was purified using a Millipore Simplicity System (EMD Millipore, Billerica, MA, USA). All other chemicals were of analytical reagent grade and used without further purification.

Beagle dogs weighing 12-15 $\mathrm{kg}$ were obtained from the Shanghai Xingang Laboratory Animal Co Ltd. (Shanghai, People's Republic of China). The animals used for experiments were treated according to protocols evaluated and approved by the Experimental Animal Ethical Committee of Fudan University.

\section{Methoxy-poly(ethylene glycol) poly(lactic acid) (MPEG-PLA) copolymer synthesis and characterization}

MPEG-PLA block copolymer was synthesized by ringopening polymerization of D,L-lactide, using stannous octoate as the catalyst and MPEG as the initiator. Briefly, $0.4 \mathrm{~g}$ of MPEG, $4 \mathrm{~g}$ of D,L-lactide, and $50 \mathrm{mg}$ of stannous octoate in dry toluene were added to a round-bottomed flask. The reactants were dried at $70^{\circ} \mathrm{C}$ under vacuum for 1 hour then polymerized at $160^{\circ} \mathrm{C}$ under vacuum for 4 hours. The cooled product was dissolved in dichloromethane and precipitated into an excess solvent mixture of ethyl ether and petroleum ether. The precipitant was then redissolved in acetone and precipitated into excess methanol. Finally, the purified MPEG-PLA copolymer was vacuum-dried at $40^{\circ} \mathrm{C}$ for 24 hours and stored in an electronic dryer. Proton nuclear 
magnetic resonance $\left({ }^{1} \mathrm{H}\right.$ NMR $)$ spectroscopy of MPEG-PLA was recorded in $\mathrm{CDCl}_{3}$ with a Varian Mercury Plus- $400 \mathrm{MHz}$ (Agilent Technologies, Santa Clara, CA, USA) apparatus operating at $400 \mathrm{MHz}$ at $25^{\circ} \mathrm{C}$. Chemical shifts in parts per million $(\delta)$ were determined using the chloroform signals at $7.26 \mathrm{ppm}$ as a reference. The integrals of the peaks corresponding to the poly(lactic acid) (PLA) methane protons ( $\delta 5.18 \mathrm{ppm})$ and the poly(ethylene glycol) (PEG) methylene protons $(\delta 3.65 \mathrm{ppm})$ were used to determine the weight ratio of PLA to PEG and to calculate the average number molecular weight (Mn) of the PLA moiety. The molecular weight and molecular distribution of the polymer were also determined by gel permeation chromatography (GPC) as previously described ${ }^{25}$ using an Agilent 1,100 GPC (Agilent Technologies) with tetrahydrofuran as the solvent.

\section{Preparation and characterization of minocycline-loaded NPs}

Preparation of minocycline-loaded NPs

Minocycline-loaded nanoparticles (MIN-NPs) were prepared using an emulsion/solvent evaporation method. In brief, $40 \mathrm{mg}$ of MPEG-PLA and $8 \mathrm{mg}$ of MIN were dissolved in $1 \mathrm{~mL}$ of dichloromethane then were added into $5 \mathrm{~mL}$ of $0.6 \%$ sodium cholate aqueous solution. The mixture was intensively emulsified by sonication (200 W, 5 seconds) 15 times in ice water using a JY92-II probe sonicator (Ningbo Scientz Biotechnology Co Ltd., Ningbo, People's Republic of China). After evaporating dichloromethane off with a RE-5205 rotary evaporator (Shanghai Yarong Biochemistry Instrument Factory, People's Republic of China) at $37^{\circ} \mathrm{C}$, the obtained NPs were concentrated by centrifugation at 21,000 $\mathrm{g}$ for 45 minutes using a TJ-25 centrifuge (Beckman Coulter Inc., Brea, CA, USA). After discarding the supernatant, the NPs were resuspended in $0.3 \mathrm{~mL}$ of deionized water. Blank NPs were prepared using the same procedure without the addition of MIN in dichloromethane.

\section{Morphology and particle size}

The particle size and zeta potential of the NPs were determined by dynamic light scattering using a Nano-ZS Malvern Zetasizer Nano analyzer (Malvern Instruments, Malvern, UK). The morphology of the NPs was observed using an H-600 transmission electron microscope (Hitachi, Tokyo, Japan) after negative staining with $2 \%$ sodium phosphotungstate solution.

\section{Drug encapsulation efficiency and loading capacity}

The drug encapsulation efficiency (EE) and drug loading capacity (LC) of the MIN-NPs was investigated as previously described. ${ }^{25}$ Briefly, the minocycline concentration in the supernatant was determined by high-performance liquid chromatography (HPLC) using a Waters ${ }^{\circledR}$ e2695 Separations Module (Waters Corporation, Milford, MA, USA) equipped with an Agilent Zorbax SB analytical column $(150 \times 4.6 \mathrm{~mm}$, pore size $5 \mu \mathrm{m}$; Agilent Technologies). The mobile phase was a mixture of methanol, acetonitrile, and $0.01 \mathrm{M}$ $\mathrm{KH}_{2} \mathrm{PO}_{4}(23: 5: 22 \mathrm{v} / \mathrm{v} / \mathrm{v})$ containing $0.03 \mathrm{mM} \mathrm{Na}{ }_{2}$ EDTA and $60 \% \mathrm{HClO}_{4}(2.9 \mathrm{~mL})$, adjusted to $\mathrm{pH} 2.5$ with $10 \mathrm{M}$ $\mathrm{KOH} .{ }^{26}$ The flow rate was $1.2 \mathrm{~mL} / \mathrm{min}$. The sample injection volume was $20 \mu \mathrm{L}$, and the detector wavelength was $350 \mathrm{~nm}$. The drug EE was calculated as $\mathrm{EE}=\left(\mathrm{MIN}_{\text {total }}-\mathrm{MIN}_{\text {supernatant }}\right) /$ $\mathrm{MIN}_{\text {total }} \times 100 \%$ and the drug LC was calculated as LC $=$ $\left(\mathrm{MIN}_{\text {total }}-\mathrm{MIN}_{\text {supernatant }}\right) /$ materials $\times 100 \%$.

\section{In vitro release study}

The in vitro release profile of minocycline from the MINNPs was investigated by a dialysis method using phosphatebuffered saline (PBS; 0.01 M, pH 7.4) as the release medium. Briefly, $1 \mathrm{~mL}$ of MIN solution or MIN-NP suspension in PBS (containing $100 \mu \mathrm{g}$ of MIN) was introduced into a dialysis bag (MWCO 8,000 Da; Greenbird Inc., Shanghai, People's Republic of China) and incubated in $10 \mathrm{~mL}$ of release medium at $37^{\circ} \mathrm{C}$ at the shaking speed of $100 \mathrm{rpm}$. At each setting time point, a $0.2 \mathrm{~mL}$ aliquot was withdrawn, and, immediately, an equal volume of fresh release medium was added. The released samples were analyzed by HPLC as already described. Samples stored were away from light throughout the experimental procedure. For the release study, these experiments were performed in quadruplicate.

\section{In vivo pharmacokinetics and pharmacodynamics}

\section{Periodontitis modeling in beagle dogs}

Periodontitis in beagle dogs was modeled by tying ligatures around the cervical region of the tooth using dental ligature wire (ClassOne Orthodontics, Carlsbad, CA, USA). ${ }^{27}$ Briefly, 2 weeks after scaling, dogs were anesthetized by intravenous injection of pentobarbital sodium at a dose of $30 \mathrm{mg} / \mathrm{kg}$. Experimental periodontitis was induced on the left upper second premolar (PM2), third premolar (PM3), and fourth premolar (PM4) as well as the left lower PM3, PM4, and first molar (PM1). Prior to ligation, the gingival attachment was incised, and the periodontal ligaments were undermined until a periodontal pocket depth of up to $3 \mathrm{~mm}$ was reached with a straight elevator (Osung MND Co Ltd., Gyeonggi-do, Korea). After undermining, a shallow notch was made in the mesial and distal region of each tooth with a round bur to act 
as a retentive groove for the ligature. After ligature placement, tramadol (4 mg/kg, intramuscular injection) was administered twice a day for 3 days for pain control. To promote plaque formation, soft moistened food was given for the following 8 weeks. The ligatures were checked weekly and any missing ligatures were replaced immediately. To evaluate periodontal status, the clinical periodontal parameters of plaque index (PI), gingival index (GI), periodontal pocket depth (PPD), clinical attachment level, and bleeding on probing were recorded. ${ }^{3}$

\section{Pharmacokinetics experiments}

Pharmacokinetics experiments were performed as previously described. ${ }^{3}$ In brief, 8 weeks after dental ligature, dogs were anesthetized by intravenous injection of pentobarbital sodium. Fifty microliters of either minocycline solution in N-methyl2-pyrrolidone (NMP) (2\%), MIN-NPs, or Periocline were injected into the periodontal pocket of each beagle dog by a disposable syringe with a 21-gauge needle. No periodontal dressing or adhesive was used to aid in retention of the minocycline formulations. At a set time point after administration, the dogs were anesthetized and GCF samples were collected by positioning an absorbent paper point (Meta, Tianjin, People's Republic of China) at the orifice of the sulcus for 30 seconds. To avoid contamination with blood and saliva, the paper strips were manipulated gently. The paper strips were weighed and the increase in weight was calculated. GCF volume was measured with a standard curve that was calibrated using bovine serum. Minocycline was immediately eluted from the paper strips by $0.1 \mathrm{M}$ glycine buffer and stored at $-80^{\circ} \mathrm{C}$. GCF minocycline levels were calculated by dividing the content of each sample pool by total volume. All GCF samples were processed with minimal delay. Minocycline concentrations in GCF were plotted with time and processed by DAS software (v 2.0; Professional Committee of Pharmacomtrics of Chinese Pharmacological Society, People's Republic of China) to calculate the pharmacokinetics parameters.

\section{Pharmacodynamics experiments}

The pharmacodynamics experiments were performed as described in the "Pharmacokinetics experiments" section with a little modification. Eight weeks after dental ligature, 12 dogs were randomly assigned to four groups: saline, free minocycline, MIN-NPs, and Periocline. After anesthetization with pentobarbital sodium, dogs were treated with $50 \mu \mathrm{L}$ of saline, minocycline solution in NMP (2\%), MIN-NPs, or Periocline by injection into the periodontal pocket of each beagle dog with a disposable syringe. The clinical periodontal parameters of PI, GI, PPD, and clinical attachment level were recorded every 3 days to evaluate the therapeutic efficacy of the MIN-NPs.

\section{Statistical analysis}

The pharmacokinetics and clinical parameters for each group are expressed as the mean \pm standard deviation. Statistical differences were determined by one-way analysis of variance, followed by Dunnett post-hoc analysis for multi-group comparison. Data within the $95 \%$ confidence level were considered significant.

\section{Results}

\section{MPEG-PLA copolymer synthesis and characterization}

Block copolymer MPEG-PLA was synthesized with molecular weight of 33,126 Da, as determined by ${ }^{1} \mathrm{H}$ NMR. The typical ${ }^{1} \mathrm{H}$ NMR spectra and chemical shifts (Figure 1) were in agreement with previously published data on MPEG-PLA copolymers. Peaks at 3.38 ppm (a), 3.65 ppm (b), $5.18 \mathrm{ppm}$ (c), and $1.59 \mathrm{ppm}$ (d) confirmed the block nature of the MPEG-PLA copolymer. GPC measurements indicated that the polydispersity index and number-based molecular weight of MPEG-PLA were 1.25 and 28,932 Da, respectively. MPEG-PLA was selected and synthesized for preparing NPs due to its biodegradability. Unlike other nonbiodegradable polymers, PEG-PLA NPs should be fully biodegradable, leaving no potentially toxic byproducts upon their degradation, after in vivo application for a long time.

\section{Preparation and characterization of MIN-NPs}

Morphology, particle size, and zeta potential

As shown in Figure 2, transmission electron microscopy showed that the minocycline-loaded PEG-PLA NPs were spherical with a uniform size and well dispersed without any adhesion or aggregation. The mean

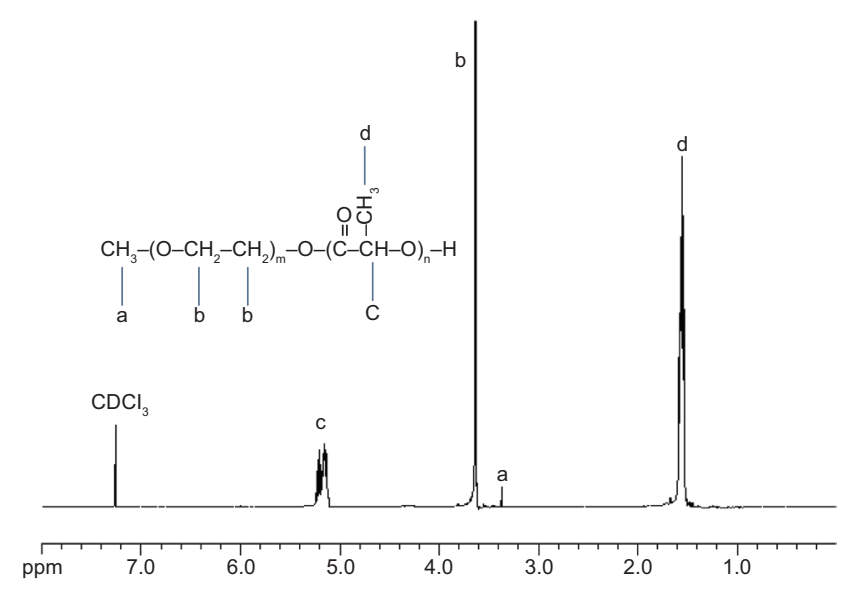

Figure I Proton nuclear magnetic resonance spectra of the MPEG-PLA in $\mathrm{CDCl}_{3}$. Abbreviation: MPEG-PLA, methoxy-poly(ethylene glycol) poly(lactic acid). 


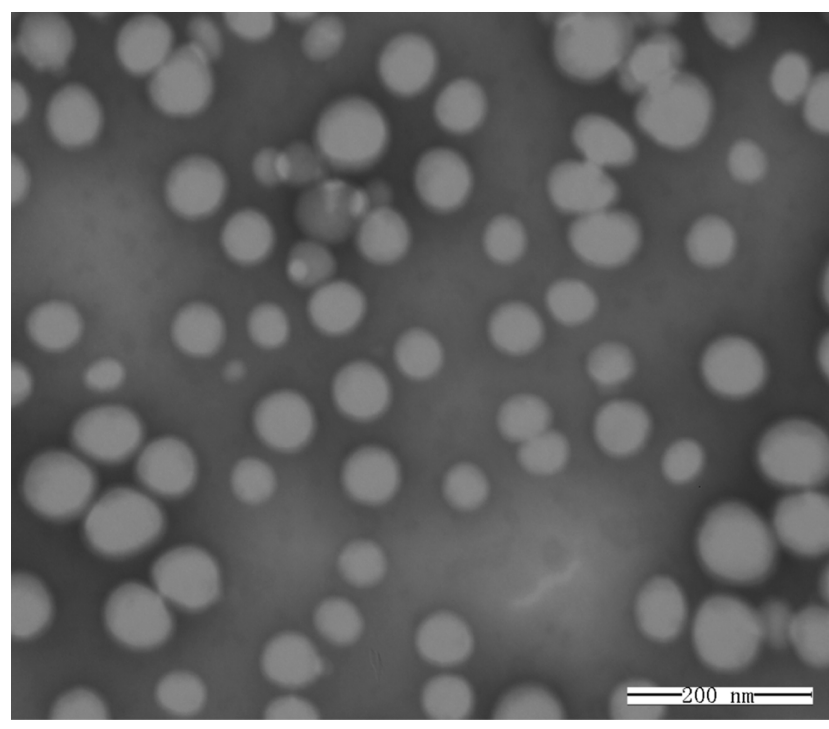

Figure 2 Transmission electron microscopy images of minocycline-loaded nanoparticles negatively stained with phosphotungstic acid solution.

Note: Scale bar $200 \mathrm{~nm}$.

\section{A}

\section{Size distribution by intensity}

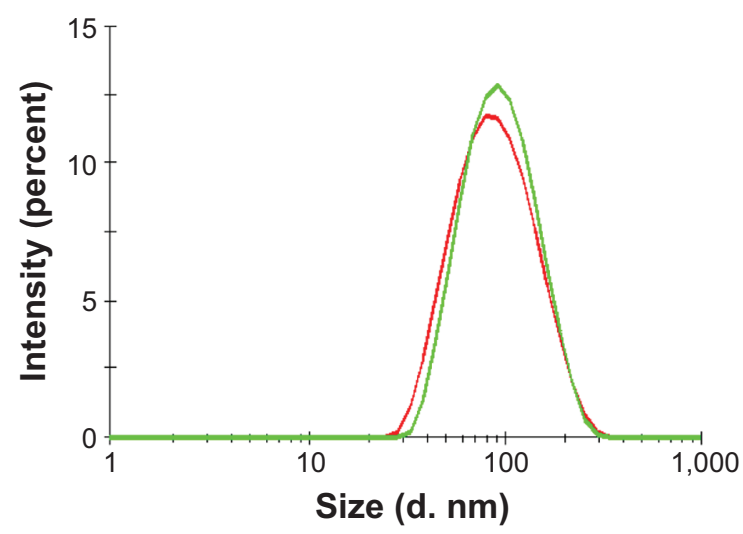

B

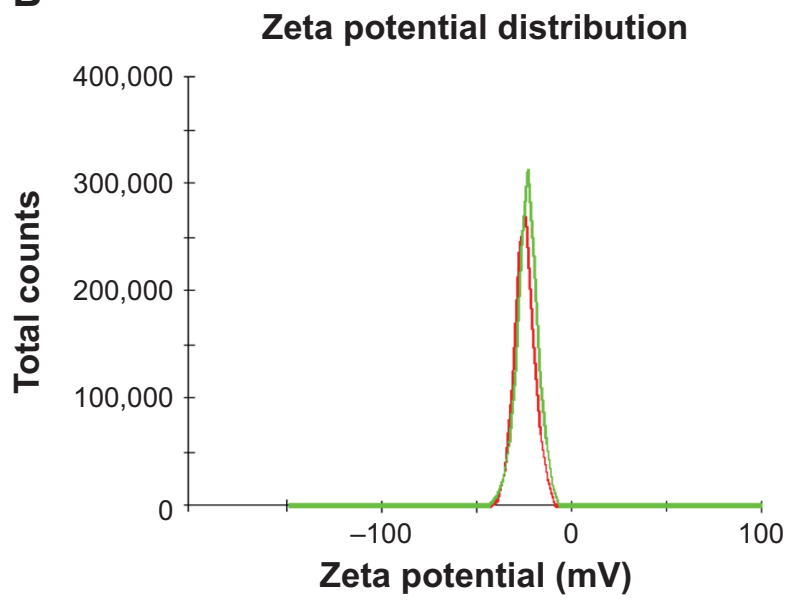

Figure 3 The particle-size distribution and surface zeta potential of minocyclineloaded nanoparticles (NPs) (green line) and blank NPs (red line). particle size of the MIN-NPs was $98 \pm 12 \mathrm{~nm}$ (Figure 3), which was slightly increased compared with that of blank NPs $(90 \pm 16 \mathrm{~nm})$. In a previous report, ${ }^{21}$ poly(lactic-co-glycolic acid) (PLGA)-based NPs were prepared using different methods, but the particle sizes of these PLGA NPs ranged from $85 \mathrm{~nm}$ to $7,070 \mathrm{~nm}$, and most of them were larger than $200 \mathrm{~nm}$. Moreover, PLGA NPs without a surface coating or PEG or another hydrophilic component are not stable and prefer to aggregate in physiological conditions. ${ }^{28}$ Therefore, the local delivery of PLGA NPs in periodontal pockets is limited. Considering the relationship between particle size and tissue absorption and penetration, smaller-size stable PEG-PLA NPs were prepared in this study. A negative surface charge was detected for the MIN-NPs ( -24 to $-20 \mathrm{mV}$ ), which was similar to that of the blank NPs $(-23$ to $-20 \mathrm{mV})$ (Figure 3). No significant changes in the zeta potential of the NPs were observed after MIN loading.

\section{Drug EE and LC}

The drug LC and EE of the MIN-NPs were detected by HPLC, and the values of LC and EE were $9.3 \% \pm 0.2 \%$ and $46.5 \% \pm 0.9 \%$, respectively. The highest LC and EE for PLGA-based NPs prepared using different methods were $1.92 \% \pm 0.19 \%$ and $29.95 \% \pm 2.47 \%$ respectively, ${ }^{21}$ and both of these two values are lower than those obtained in our study, indicating the superiority of our MIN-NP design.

\section{In vitro release of MIN-NPs}

The release profiles of minocycline solution and MIN-NPs in PBS (0.01 M, pH $=7.4)$ at $37^{\circ} \mathrm{C}$ showed that almost $100 \%$ of the minocycline solution was released within 24 hours, while the cumulative release of MIN from the MIN-NPs

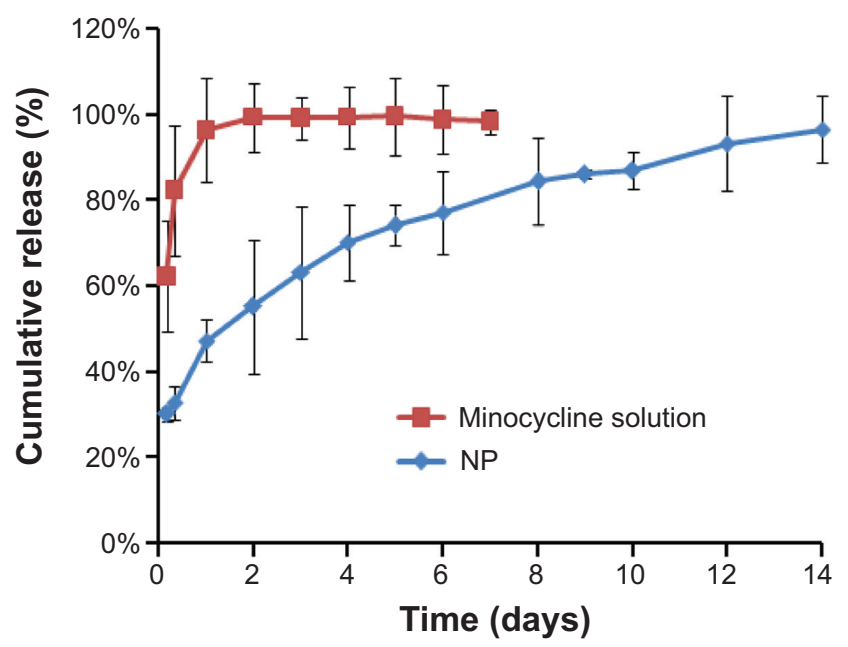

Figure 4 The releasing curve of minocycline hydrochloride from minocyclineloaded nanoparticles (NPs) in phosphate-buffered saline $(0.01 \mathrm{M}, \mathrm{pH}=7.4)$ at $37^{\circ} \mathrm{C}$. Notes: Data are presented as the mean \pm standard deviation; $n=4$. 


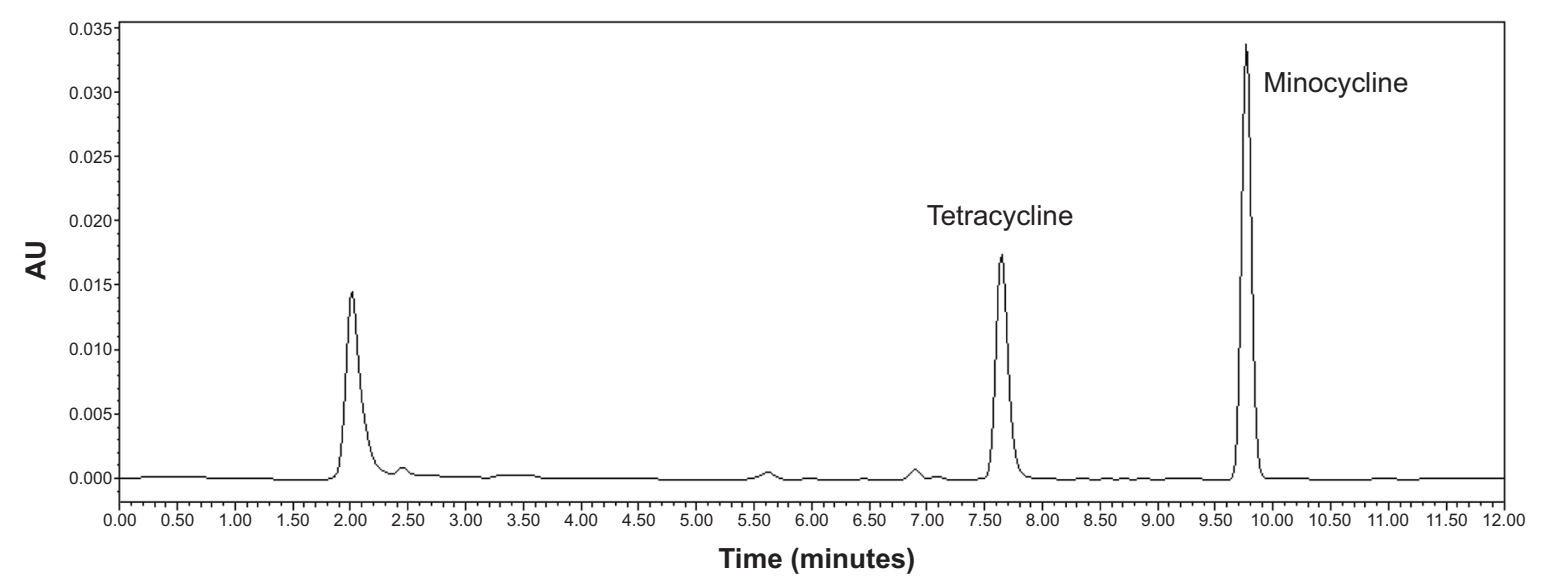

Figure 5 A typical high-performance liquid chromatography figure of a gingival crevice fluid sample.

was approximate $96 \%$ after 14 days (Figure 4), which demonstrated the marked sustained-release characteristics of the MIN-NPs. Qin et al prepared a kind of tinidazole in situ forming PLA implant, and the release of tinidazole for local delivery was about 7 days. ${ }^{3}$ The longest release time obtained with Elyzol was 48 hours. ${ }^{29}$ Compared with these results, the release of MIN-NPs over 14 days demonstrated a good sustained-release property. The cumulative release of MIN from the MIN-NPs and the release time were fitted with a first-order drug-releasing equation $(\ln (1-\mathrm{Q})=-\mathrm{Kt})$, the Higuchi equation $\left(\mathrm{Q}=\mathrm{Kt}^{1 / 2}\right)$, and Ritger-Peppas equation $\left(\mathrm{Q}=\mathrm{K} \mathrm{t}^{\mathrm{n}}\right)$, and the best result $\left(\mathrm{Q}=0.47 \mathrm{t}^{0.27}, r=0.998\right)$ was achieved with Ritger-Peppas equation, indicating the release of MIN mainly depended on diffusion.

\section{Pharmacokinetics of different formulations of MIN in GCF}

The concentration of MIN in GCF was determined by HPLC, and the baseline separation of MIN and tetracycline was achieved with the chromatographic conditions outlined. The retention time (RT) for MIN and tetracycline was 7.768 minutes and 9.801 minutes, respectively (Figure 5). The standard curve $\left(\mathrm{y}=0.158 \mathrm{x}+0.359, R^{2}=0.997\right)$ was obtained by the line regression of MIN concentration with the peak area ratio of MIN and tetracycline, while the linearity was given in the range of $0.1-20.0 \mu \mathrm{g} / \mathrm{mL}$.

The pharmacokinetics curve of MIN in GCF (Figure6) showed that the concentration of MIN in GCF declined rapidly after the administration of MIN solution and was lower than the median effective concentration $(1 \mu \mathrm{g} / \mathrm{mL})$ after 2 days. The concentration of MIN in GCF declined slowly after administration of Periocline, and the action of the drug lasted 8 days $(>1 \mu \mathrm{g} / \mathrm{mL})$, suggesting good sustained release of Periocline. However, after administration of the MIN-NPs, the concentration of MIN in GCF declined slowly and was still higher than $1 \mu \mathrm{g} / \mathrm{mL}(1.28 \mu \mathrm{g} / \mathrm{mL})$ 12 days later, representing the longest action time among three groups. The long action time of the MIN-NPs may be related to either the drug's sustained release or the adherence and penetration of MIN-NPs into inflammation tissues. The pharmacokinetics parameters of MIN in GCF, as calculated using DAS pharmacokinetics software, are shown in Table 1. The elimination of MIN in each group was well fitted to two-compartment open models. There were significant differences in the area under the curve (AUC) from zero to infinity $(0-\infty)$, mean retention time (MRT) $(0-\infty)$, half-life $\left(\mathrm{t}_{1 / 2}\right)$, elimination rate constant $(\mathrm{k})$, and clearance $(\mathrm{Cl})$ between groups $(P<0.01)$. The $\mathrm{AUC}_{(0-\infty)}, \mathrm{MRT}_{(0-\infty)}$, and $\mathrm{t}_{1 / 2}$ of the MIN-NP group were 4.62-fold, 2.21-fold, and 3.74-fold, respectively, those of the MIN solution group. The $\mathrm{AUC}_{(0-\infty)}$ and $\mathrm{MRT}_{(0-\infty)}$ of the MIN-NP group were 1.42-fold and 1.21-fold, respectively, higher than those of the Periocline group, indicating higher local bioavailability and the better effect of the former.

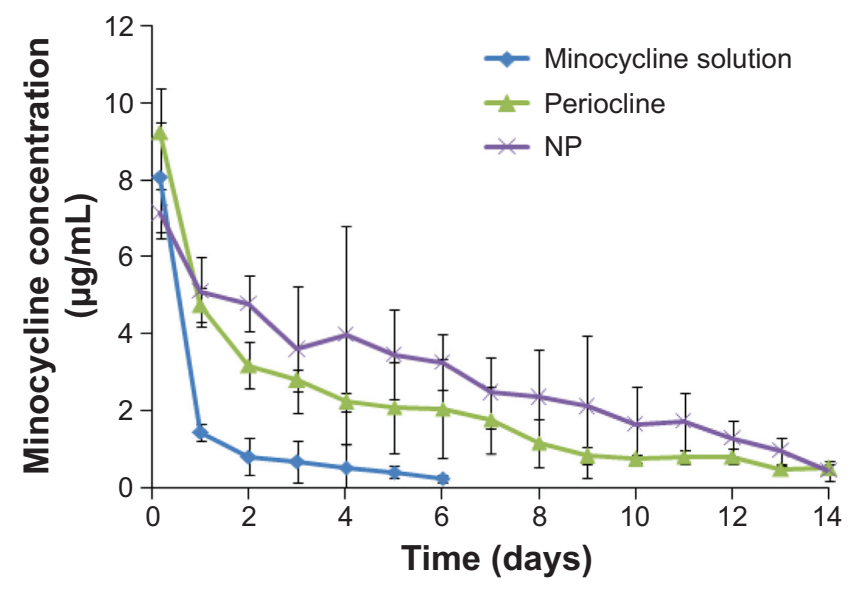

Figure 6 Pharmacokinetics curve of minocycline hydrochloride (MIN) in gingival crevice fluid after local administration of different MIN formulations. Notes: Data presented as the mean \pm standard deviation; $\mathrm{n}=6$. 
Table I Pharmacokinetics parameters of minocycline hydrochloride (MIN) in gingival crevice fluid after local administration of different MIN formulations $(n=6)$

\begin{tabular}{|c|c|c|c|}
\hline Parameter & MIN-NPs & Periocline $^{\circledR}$ & MIN solution \\
\hline $\mathrm{AUC}_{(0-\mathrm{t})}(\mathrm{mg} / \mathrm{L} * \mathrm{~d})$ & $40.060 \pm 4.120^{a, b}$ & $28.140 \pm 3.350^{b}$ & $7.890 \pm 1.200$ \\
\hline $\operatorname{AUC}_{(0-\infty)}\left(\mathrm{mg} / \mathrm{L}^{*} \mathrm{~d}\right)$ & $42.470 \pm 4.530^{\mathrm{a}, \mathrm{b}}$ & $32.220 \pm 3.630^{\mathrm{b}}$ & $9.190 \pm 1.420$ \\
\hline $\mathrm{MRT}_{(0-t)}^{(d)}$ & $4.990 \pm 0.520^{b}$ & $4.130 \pm 0.420^{b}$ & $1.330 \pm 0.150$ \\
\hline $\mathrm{MRT}_{(0-\infty)}(\mathrm{d})$ & $5.690 \pm 0.630^{b}$ & $6.490 \pm 0.690^{b}$ & $2.570 \pm 0.290$ \\
\hline $\mathrm{t}_{1 / 2}(\mathrm{~d})$ & $3.460 \pm 0.360^{\mathrm{a}, \mathrm{b}}$ & $2.860 \pm 0.290^{b}$ & $0.920 \pm 0.100$ \\
\hline$k\left(d^{-1}\right)$ & $0.20 I \pm\left. 0.02\right|^{\mathrm{a}, \mathrm{b}}$ & $0.242 \pm 0.025^{b}$ & $0.750 \pm 0.084$ \\
\hline $\mathrm{T}_{\max }(\mathrm{d})$ & 0.167 & 0.167 & 0.167 \\
\hline $\mathrm{V}(\mathrm{L})$ & $0.124 \pm 0.025^{\mathrm{a}}$ & $0.147 \pm 0.029$ & $0.169 \pm 0.015$ \\
\hline $\mathrm{Cl}(\mathrm{L} / \mathrm{d})$ & $0.025 \pm 0.005^{\mathrm{a}, \mathrm{b}}$ & $0.036 \pm 0.007^{b}$ & $0.127 \pm 0.011$ \\
\hline $\mathrm{C}_{\max }(\mathrm{mg} / \mathrm{L})$ & $7.130 \pm 1.220^{\mathrm{a}}$ & $9.230 \pm 1.350$ & $8.060 \pm 1.390$ \\
\hline
\end{tabular}

Notes: Data are presented as the mean \pm standard deviation; ${ }^{a}<<0.05$ vs Periocline; b $P<0.01$ vs $M I N$ solution.

Abbreviations: $0-\infty$, time zero to infinity; $0-t$, zero to time $t$; $A \cup C$, area under the curve; $\mathrm{Cl}$, clearance; $\mathrm{C}_{\max }$, peak concentration; $\mathrm{k}$, elimination rate constant; $\mathrm{MIN}$ NPs, minocycline-loaded nanoparticles; MRT, mean retention time; $t_{1 / 2}$, half-life; $T_{\text {max }}$, time to peak concentration; $\mathrm{V}$, apparent volume of distribution; $d$, days.

\section{Pharmacodynamics of MIN-NPs}

As shown in Figure 7, there were no significant differences in clinical periodontal parameters between the MIN solution group and saline group after the local delivery of MIN

\section{A}

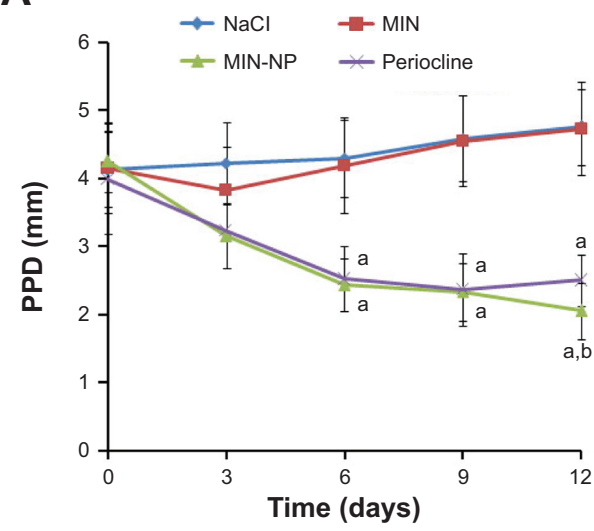

C

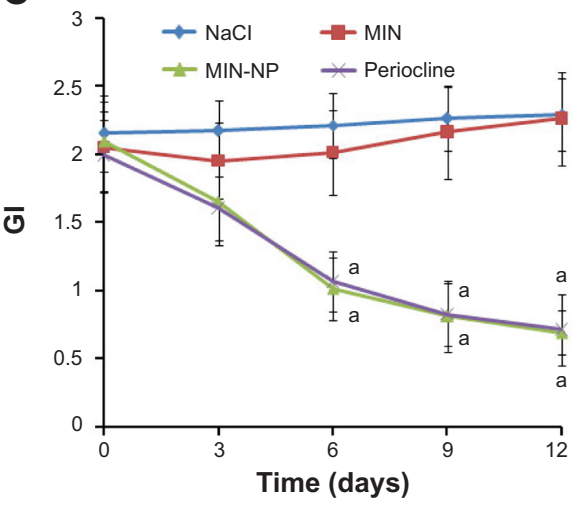

solution in the dog periodontitis models, indicating the MIN solution had no obvious treatment efficacy for periodontitis. However, for Periocline and MIN-NPs, 6 days after local administration, clinical periodontal parameters (PPD, PI, GI) were significantly improved, and significantly better than those of the MIN solution group. In addition, 9 days after administration, the PPD, PI, and bleeding on probing of the Periocline group increased again, which may be because an effective drug concentration of Periocline could only be maintained for 8 days (Figure 6). Twelve days after the administration, the PPD and PI of the MIN-NP group were still significantly lower than those of the MIN solution and Periocline groups, suggesting that MIN has a long treatment efficacy, which agreed well with pharmacokinetics results.

\section{Conclusion}

In order to maintain the effective concentration of MIN in GCF for a longer period, MIN-NPs were prepared for evaluation in the study. Different forms of MIN were placed into the periodontal pockets of beagle dogs with periodontitis. GCF samples were collected after drug delivery and quantified by HPLC. After local administration of MIN-NPs, the

B

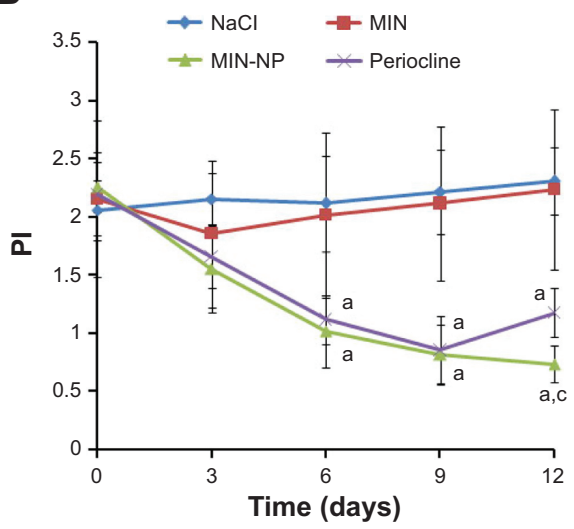

D

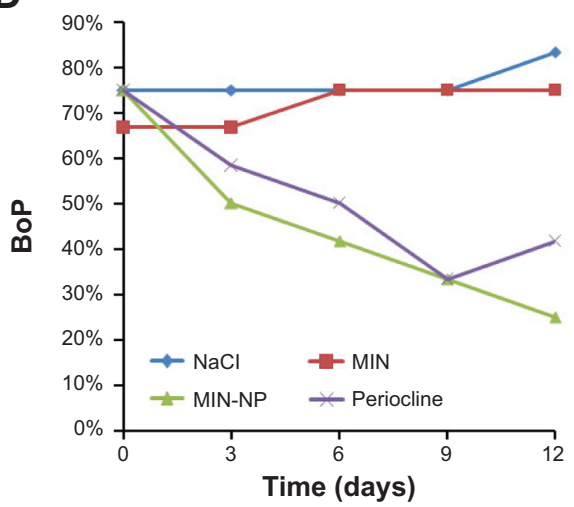

Figure 7 Changes in clinical periodontitis parameters after local administration of different minocycline hydrochloride (MIN) formulations. Notes: Data presented as the mean \pm standard deviation; $n=6$. ${ }^{a} P<0.01$ vs $M I N$ solution; ${ }^{b} P<0.05,{ }^{c} P<0.01$ vs $P$ eriocline ${ }^{\circledR}$.

Abbreviations: BoP, bleeding on probing; GI, gingival index; MIN-NP, minocycline-loaded nanoparticles; PI, plaque index; PPD, periodontal pocket depth. 
minocycline concentration in GCF decreased slowly and an effective drug concentration was retained for a longer time than with Periocline. The MIN-NPs demonstrated anti-periodontitis effects that MIN-NPs could significantly decrease the symptoms of periodontitis, compared with Periocline and MIN solution. These findings suggest that MIN-NPs might have great potential in the treatment of periodontitis.

\section{Acknowledgments}

This work was supported by the Shanghai Municipal Public Health Bureau Research Grant, National Science and Technology Major Project no. 2012ZX09304004, National Natural Science Foundation of China no. 81001404, the Doctoral Fund of the Ministry of Education of China no. 20100071120050, and the Zhuoxue Plan of Fudan University.

\section{Disclosure}

The authors declare no conflicts of interest in this work.

\section{References}

1. Socransky SS, Haffajee AD. Evidence of bacterial etiology: a historical perspective. Periodontol 2000. 1994;5:7-25.

2. Armitage GC. Development of a classification system for periodontal diseases and conditions. Ann Periodontol. 1999;4(1):1-6.

3. Qin Y, Yuan M, Li L, Li W, Xue J. Formulation and evaluation of in situ forming PLA implant containing tinidazole for the treatment of periodontitis. J Biomed Mater Res B Appl Biomater. 2012;100(8): 2197-2202.

4. Tezel A, Yucel O, Orbak R, et al. The gingival crevicular fluid ciprofloxacin level in subjects with gingivitis and periodontitis, and its effects on clinical parameters. J Periodontal Res. 2005;40(5):395-400.

5. Haffajee AD, Socransky SS. Microbial etiological agents of destructive periodontal diseases. Periodontol 2000. 1994;5:78-111.

6. Slots J, Chen C. The oral microflora and human periodontal disease. In: Tannock GW, editor. Medical Importance of the Normal Microflora. Dordrecht: Kluwer; 1999:101-127.

7. Marsh PD. Dental plaque: biological significance of a biofilm and community life-style. J Clin Periodontol. 2005;32(Suppl 6):7-15.

8. Scannapieco FA, Dasanayake AP, Chhun N. "Does periodontal therapy reduce the risk for systemic diseases?" Dent Clin North Am. 2010;54(1):163-181.

9. Apatzidou DA, Kinane DF. Nonsurgical mechanical treatment strategies for periodontal disease. Dent Clin North Am. 2010;54(1):1-12.

10. Lu SH, Huang RY, Chou TC. Magnolol ameliorates ligature-induced periodontitis in rats and osteoclastogenesis: in vivo and in vitro study. Evid Based Complement Alternat Med. 2013;2013: Article ID 634095.

11. van Winkelhoff AJ, Rams TE, Slots J. Systemic antibiotic therapy in periodontics. Periodontol 2000. 1996;10:45-78.

International Journal of Nanomedicine

\section{Publish your work in this journal}

The International Journal of Nanomedicine is an international, peerreviewed journal focusing on the application of nanotechnology in diagnostics, therapeutics, and drug delivery systems throughout the biomedical field. This journal is indexed on PubMed Central,

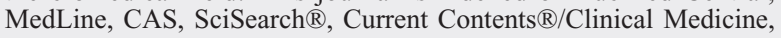

12. van Winkelhoff AJ, Herrera Gonzales D, Winkel EG, Dellemijn-Kippuw N, Vandenbroucke-Grauls CM, Sanz M. Antimicrobial resistance in the subgingival microflora in patients with adult periodontitis. A comparison between The Netherlands and Spain. J Clin Periodontol. 2000;27(2):79-86.

13. Kelly HM, Deasy PB, Ziaka E, Claffey N. Formulation and preliminary in vivo dog studies of a novel drug delivery system for the treatment of periodontitis. Int J Pharm. 2004;274(1):167-183.

14. Jain N, Jain GK, Javed S, et al. Recent approaches for the treatment of periodontitis. Drug Discov Today. 2008;13(21):932-943.

15. Kenawy el-R, Bowlin GL, Mansfield K, et al. Release of tetracycline hydrochloride from electrospun poly(ethylene-co-vinylacetate), poly(lactic acid), and a blend. J Control Release. 2002;81(1-2):57-64.

16. Tonetti MS, Pini-Prato G, Cortellini P. Principles and clinical applications of periodontal controlled drug delivery with tetracycline fibers. Int J Periodontics Restorative Dent. 1994;14(5):421-435.

17. Kamaly N, Xiao Z, Valencia PM, Radovic-Moreno AF, Farokhzad OC. Targeted polymeric therapeutic nanoparticles: design, development and clinical translation. Chem Soc Rev. 2012;41(7):2971-3010.

18. Rose S, Prevoteau A, Elzière P, Hourdet D, Marcellan A, Leibler L. Nanoparticle solutions as adhesives for gels and biological tissues. Nature. 2014;505(7483):382-385.

19. Piñón-Segundo E, Ganem-Quintanar A, Alonso-Pérez V, QuintanarGuerrero D. Preparation and characterization of triclosan nanoparticles for periodontal treatment. Int J Pharm. 2005;294(1):217-232.

20. Chwalibog A, Sawosz E, Hotowy A, et al. Visualization of interaction between inorganic nanoparticles and bacteria or fungi. Int J Nanomedicine. 2010;5:1085-1094.

21. Kashi TSJ, Eskandarion S, Esfandyari-Manesh M, et al. Improved drug loading and antibacterial activity of minocycline-loaded PLGA nanoparticles prepared by solid/oil/water ion pairing method. Int J Nanomedicine. 2012;7:221-234.

22. Liu D, Yang PS. Minocycline hydrochloride nanoliposomes inhibit the production of TNF- $\alpha$ in LPS-stimulated macrophages. Int J Nanomedicine. 2012;7:4769-4775.

23. Gopinath V, Ramakrishnan T, Emmadi P, Ambalavanan N, Mammen B, Vijayalakshmi. Effect of a controlled release device containing minocycline microspheres on the treatment of chronic periodontitis: a comparative study. J Indian Soc Periodontol. 2009;13(2):79-84.

24. Kulkarni RK, Pani KC, Neuman C, Leonard F. Polylactic acid for surgical implants. Arch Surg. 1966;93(5):839-843.

25. Pang Z, Gao H, Yu Y, et al. Brain delivery and cellular internalization mechanisms for transferrin conjugated biodegradable polymersomes. Int J Pharm. 2011;415(1-2):284-292.

26. Colovic M, Caccia S. Liquid chromatographic determination of minocycline in brain-to-plasma distribution studies in the rat. $J$ Chromatogr B Analyt Technol Biomed Life Sci. 2003;791(1-2):337-343.

27. Kim SE, Lee ER, Lee Y, et al. A modified method for inducing periodontitis in dogs using a silk-wire twisted ligature. J Vet Sci. 2012; 13(2): 193-197.

28. Gref R, Minamitake Y, Peracchia MT, Trubetskoy V, Torchilin V, Langer R. Biodegradable long-circulating polymeric nanospheres. Science. 1994;263(5153):1600-1603.

29. Norling T, Lading P, Engström S, Larsson K, Krog N, Nissen SS. Formulation of a drug delivery system based on a mixture of monoglycerides and triglycerides for use in the treatment of periodontal disease. J Clin Periodontol. 1992;19(9):687-692.

Journal Citation Reports/Science Edition, EMBase, Scopus and the Elsevier Bibliographic databases. The manuscript management system is completely online and includes a very quick and fair peer-review system, which is all easy to use. Visit http://www.dovepress.com/ testimonials.php to read real quotes from published authors. 